\title{
Electromagnetic finite-size effects beyond the point-like approximation
}

\author{
Matteo Di Carlo ${ }^{1, *}$, Maxwell T. Hansen ${ }^{1, * *}$, Nils Hermansson-Truedsson ${ }^{2, * * *}$, and Antonin \\ Portelli ${ }^{1, * * * *}$ \\ ${ }^{1}$ Higgs Centre for Theoretical Physics, School of Physics and Astronomy, University of Edinburgh, \\ Peter Guthrie Tait Road, Edinburgh, EH9 3FD, United Kingdom \\ ${ }^{2}$ Albert Einstein Center for Fundamental Physics, Institute for Theoretical Physics, Universität Bern, \\ Sidlerstrasse 5, 3012 Bern, Switzerland
}

\begin{abstract}
We present a model-independent and relativistic approach to analytically derive electromagnetic finite-size effects beyond the point-like approximation. The key element is the use of electromagnetic Ward identities to constrain vertex functions, and structure-dependence appears via physical form-factors and their derivatives. We apply our general method to study the leading finitesize structure-dependence in the pseudoscalar mass (at order $1 / L^{3}$ ) as well as in the leptonic decay amplitudes of pions and kaons (at order $1 / L^{2}$ ). Knowledge of the latter is essential for Standard Model precision tests in the flavour physics sector from lattice simulations.
\end{abstract}

\footnotetext{
*e-mail: matteo.dicarlo@ed.ac.uk

**e-mail: maxwell.hansen@ed.ac.uk

***Speaker, e-mail: nils@itp.unibe.ch

****e-mail: antonin.portelli@ed.ac.uk
} 


\section{Introduction}

Lattice quantum chromodynamics (QCD) allows for systematically improvable Standard Model (SM) precision tests from numerical simulations performed in a finite-volume (FV), discretised Euclidean spacetime. In order to reach (sub-)percent precision in lattice predictions, also strong and electromagnetic isospin breaking corrections have to be included. The latter are encoded via quantum electrodynamics (QED), but the inclusion of QED in a FV spacetime is complicated because of Gauss' law [1]. This problem is related to zeromomentum modes of photons and the absence of a QED mass-gap. Several prescriptions of how to include QED in a finite volume have been formulated and the one used here is $\mathrm{QED}_{\mathrm{L}}$ where the spatial zero-modes are removed on each time-slice. The long-range nature of QED in addition enhances the FV effects (FVEs), which typically leads to power-law FVEs that are larger than the exponentially suppressed ones for single-particle matrix elements in QCD alone.

The FVEs for a QCD+QED process depend on properties of the involved particles, including masses and charges, but also structure-dependent quantities such as electromagnetic form-factors and their derivatives. In order to analytically capture the finite-volume scaling fully, one cannot neglect hadron structure, and in the following we develop a relativistic and model-independent method to go beyond the point-like approximation at order $e^{2}$ in $\mathrm{QED}_{\mathrm{L}}$.

We consider a space-time with periodic spatial extents $L$ but with infinite time-extent. To exemplify the method, we first consider the pseudoscalar mass in Sec. 2, and then proceed to leptonic decays in Sec. 3. The discussion is based on the results in Ref. [2], and the reader is referred there for further technical details.

\section{Pseudoscalar Mass}

To study the finite-size scaling in the mass $m_{P}(L)$ of a charged hadronic spin- 0 particle $P$, we first define the full QCD+QED infinite-volume two-point Euclidean correlation function

$$
C_{2}^{\infty}(p)=\int d^{4} x\left\langle 0\left|\mathrm{~T}\left[\phi(x) \phi^{\dagger}(0)\right]\right| 0\right\rangle e^{-i p x} .
$$

Here $\phi$ is an interpolating operator coupling to $P$, and $p=\left(p_{0}, \mathbf{p}\right)$ is the momentum. We denote the finite-volume counterpart of this correlator $C_{2}^{L}(p)$, but for the moment only consider $C_{2}^{\infty}(p)$. This can be diagrammatically represented as

$$
C_{2}^{\infty}(p)=\phi=Z_{P} \cdot D(p) \cdot Z_{P}, \quad D(p)=\frac{Z\left(p^{2}\right)}{p^{2}+m_{P}^{2}}, \quad Z_{P}=\langle 0|\phi(0)| P, \mathbf{p}\rangle,
$$

where the double-line represents the QCD+QED propagator $D(p)$, the $\phi$-blob is the overlap between $\phi$ and $P$ and $Z\left(p^{2}\right)=1+O\left(p^{2}+m_{P}^{2}\right)$ is the residue of the propagator. Expanding $C_{2}^{\infty}(p)$ in (2) around $e=0$ yields

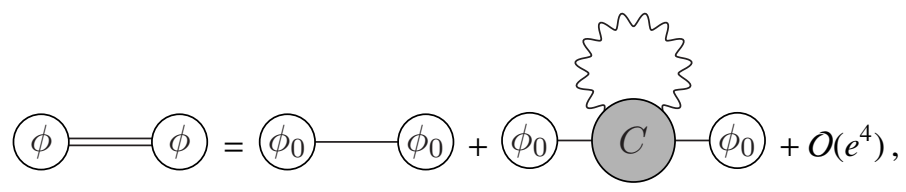


where quantities with subscript 0 are evaluated in QCD alone. The grey blob is the Compton scattering kernel defined via

$$
-C \int^{3}=C_{\mu v}(p, k, q)=\int d^{4} x d^{4} y d^{4} z e^{i p z+i k x+i q y} \frac{\left\langle 0\left|\mathrm{~T}\left[\phi(0) J_{\mu}(x) J_{v}(y) \phi^{\dagger}(z)\right]\right| 0\right\rangle}{Z_{P, 0}^{2} D_{0}(p) D_{0}(p+k+q)}
$$

Here $k$ and $q$ are incoming photon momenta and $J_{\mu}(x)$ is the electromagnetic current. Note that the unphysical dependence on the arbitrary interpolating operator $\phi$ must cancel for any physical quantity, and when the external legs in $C_{\mu v}(p, k, q)$ go on-shell the kernel is nothing but the physical forward Compton scattering amplitude. Using (3) the electromagnetic massshift of the meson is readily obtained in terms of an integral over the photon loop-momentum $k$. One may follow an equivalent procedure for the finite-volume correlation function $C_{2}^{L}(p)$, where the integral over the spatial momentum $\mathbf{k}$ is replaced by a sum. The leading electromagnetic FVEs in the mass, $\Delta m_{P}^{2}(L)$, are thus given by the sum-integral difference

$$
\Delta m_{P}^{2}(L)=-\left.\frac{e^{2}}{2} \lim _{p_{0}^{2} \rightarrow-m_{P}^{2}}\left(\frac{1}{L^{3}} \sum_{\mathbf{k}}^{\prime}-\int \frac{d^{3} \mathbf{k}}{(2 \pi)^{3}}\right) \int \frac{d k_{0}}{2 \pi} \frac{C_{\mu \mu}(p, k,-k)}{k^{2}}\right|_{\mathbf{p}=0}
$$

where the rest-frame $\mathbf{p}=0$ was chosen for convenience and the primed sum indicates the omission of the photon zero-mode $\mathbf{k}=0$ in $\mathrm{QED}_{\mathrm{L}}$. The analytical dependence on $1 / \mathrm{L}$ including structure-dependence can now be obtained from this formula through a soft-photon expansion of the integrand, i.e. an expansion order by order in $|\mathbf{k}|$ which is directly related to the expansion in $1 / L$ via $|\mathbf{k}|=2 \pi|\mathbf{n}| / L$ where $\mathbf{n}$ is a vector of integers. The first step is to decompose $C_{\mu \nu}(p, k, q)$ into two irreducible electromagnetic vertex functions $\Gamma_{1}$ and $\Gamma_{2}$ according to

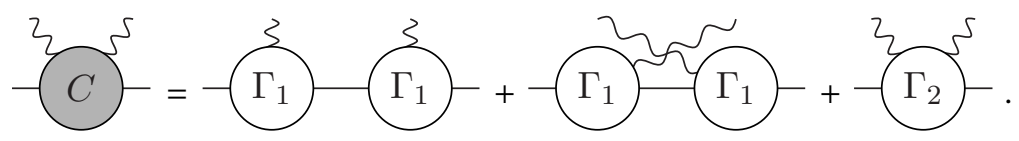

The vertex functions depend in general on the structure of the particle, as can be seen from e.g. the form-factor decomposition

$$
\Gamma_{1}=\Gamma_{\mu}(p, k)=(2 p+k)_{\mu} F\left(k^{2},(p+k)^{2}, p^{2}\right)+k_{\mu} G\left(k^{2},(p+k)^{2}, p^{2}\right),
$$

where $F\left(k^{2},(p+k)^{2}, p^{2}\right)$ and $G\left(k^{2},(p+k)^{2}, p^{2}\right)$ are structure-dependent electromagnetic formfactors depending on three virtualities. This means that $F$ and $G$ contain off-shell effects, but we stress that these non-physical quantities always cancel in the FVEs. The cancellation occurs since the vertex functions $\Gamma_{1,2}$ are related to each other and the propagator $D_{0}(p)$ via Ward identities. An example of an off-shell relation is $F\left(0, p^{2},-m_{P, 0}^{2}\right)=Z_{0}\left(p^{2}\right)^{-1}$. The derivatives of $Z_{0}\left(p^{2}\right)$ are already known in the literature as $\delta D^{(n)}(0)$ [3] and $z_{n}$ [4], but these could in principle be set to zero as they always cancel in the final results. The Ward identities further yield $G$ as a function of $F$. The form-factor $F$ also contains physical information, and for our purposes it suffices to know that $F^{(1,0,0)}\left(0,-m_{P, 0}^{2},-m_{P, 0}^{2}\right)=F^{\prime}(0)=-\left\langle r_{P}^{2}\right\rangle / 6$, where $\left\langle r_{P}^{2}\right\rangle$ is the physical electromagnetic charge radius of $P$ which is well-known experimentally [5].

Using our definitions of the vertex functions in $C_{\mu v}(p, k, q)$ in (5) we obtain the FVEs

$$
\Delta m_{P}^{2}(L)=e^{2} m_{P}^{2}\left\{\frac{c_{2}}{4 \pi^{2} m_{P} L}+\frac{c_{1}}{2 \pi\left(m_{P} L\right)^{2}}+\frac{\left\langle r_{P}^{2}\right\rangle}{3 m_{P} L^{3}}+\frac{C}{\left(m_{P} L\right)^{3}}+O\left[\frac{1}{\left(m_{P} L\right)^{4}}\right]\right\}
$$


where the $c_{j}$ are finite-volume coefficients specific to $\mathrm{QED}_{\mathrm{L}}$ arising from the sum-integral difference in (5). These are discussed in detail in Ref. [2]. Here we see the charge radius $\left\langle r_{P}^{2}\right\rangle$ appearing at order $1 / L^{3}$ and its coefficient agrees with that derived within non-relativistic scalar QED [6]. However, there is an additional structure-dependent term $C$ related to the branch-cut of the forward, on-shell Compton amplitude. This contribution can be found, in other forms, also in Refs. [3, 7], and only arises because of the $\mathrm{QED}_{\mathrm{L}}$ prescription with the subtracted zero-mode. Its value is currently unknown but one can show $C>0$ [2], meaning that it cannot cancel the charge radius contribution. Note that all unphysical offshell contributions from the form-factors $F$ and $G$ have vanished.

\section{Leptonic Decays}

Leptonic decay rates of light mesons are of the form $P^{-} \rightarrow \ell^{-} \bar{v}_{\ell}$, where $P$ is a pion or kaon, $\ell$ a lepton and $v_{\ell}$ the corresponding neutrino. These are important for the extraction of the Cabibbo-Kobayashi-Maskawa matrix elements $\left|V_{u s}\right|$ and $\left|V_{u d}\right|[8,9]$. The leading virtual electromagnetic correction to this process yields an infrared (IR) divergent decay rate $\Gamma_{0}$. One must therefore add the real radiative decay rate $\Gamma_{1}(\Delta E)$ for $P^{-} \rightarrow \ell^{-} \bar{v}_{\ell} \gamma$, where the photon has energy below $\Delta E$, to cancel the IR-divergence in $\Gamma_{0}$. The IR-finite inclusive decay rate is thus $\Gamma\left(P^{-} \rightarrow \ell^{-} v_{\ell}[\gamma]\right)$, and following the lattice procedure first laid out in Ref. [8] we may write

$$
\Gamma_{0}+\Gamma_{1}\left(\Delta E_{\gamma}\right)=\lim _{L \rightarrow \infty}\left[\Gamma_{0}(L)-\Gamma_{0}^{\mathrm{uni}}(L)\right]+\lim _{L \rightarrow \infty}\left[\Gamma_{0}^{\mathrm{uni}}(L)+\Gamma_{1}\left(L, \Delta E_{\gamma}\right)\right]
$$

Here, Ref. [8] chose to add and subtract the universal finite-volume decay rate $\Gamma^{\mathrm{uni}}(L)$, calculated in point-like scalar QED in Ref. [4], to cancel separately the IR-divergences in $\Gamma_{0}$ and $\Gamma_{1}$. In the following we are interested in only the first term in brackets. The subtracted term $\Gamma_{0}^{\text {uni }}(L)$ cancels the FVEs in $\Gamma_{0}(L)$ through order $1 / L$, and hence $\Gamma_{0}(L)-\Gamma_{0}^{\text {uni }}(L) \sim O\left(1 / L^{2}\right)$. Structure-dependence enters at order $1 / L^{2}$. With the goal of systematically improving the finite-volume scaling order by order including structure-dependence, we replace the universal contribution by

$$
\Gamma_{0}^{\mathrm{uni}}(L) \longrightarrow \Gamma_{0}^{(n)}(L)=\Gamma_{0}^{\mathrm{uni}}(L)+\sum_{j=2}^{n} \Delta \Gamma_{0}^{(j)}(L),
$$

where $n \geq 2$ and $\Delta \Gamma_{0}^{(j)}(L)$ contains the FVEs at order $1 / L^{j}$. This means that the finite-volume residual instead scales as $\Gamma_{0}(L)-\Gamma_{0}^{(n)}(L) \sim O\left(1 / L^{n+1}\right)$. We may parametrise $\Gamma_{0}^{(n)}(L)$ in terms of a finite-volume function $Y^{(n)}(L)$ according to

$$
\Gamma_{0}^{(n)}(L)=\Gamma_{0}^{\text {tree }}\left[1+2 \frac{\alpha}{4 \pi} Y^{(n)}(L)\right]+O\left(\frac{1}{L^{n+1}}\right)
$$

where $\Gamma_{0}^{\text {tree }}$ is the tree-level decay rate.

Since we are interested in the leading structure-dependent contribution we consider $Y^{(2)}(L)$. In order to derive it, we define the QCD+QED correlation function

$$
C_{W}^{r s}\left(p, p_{\ell}\right)=\int d^{4} z e^{i p z}\left\langle\ell^{-}, \mathbf{p}_{\ell}, r ; v_{\ell}, \mathbf{p}_{v_{\ell}}, s\left|\mathrm{~T}\left[O_{W}(0) \phi^{\dagger}(z)\right]\right| 0\right\rangle
$$

where $p_{\ell}=\left(p_{\ell}^{0}, \mathbf{p}_{\ell}\right)$ is the momentum of the on-shell lepton of mass $m_{\ell}, p_{v_{\ell}}=\left(p_{v_{\ell}}^{0}, \mathbf{p}_{v_{\ell}}\right)$ is the momentum of the massless neutrino and $O_{W}(0)$ is the four-fermion operator of the decay in 
question. We may diagrammatically represent this in a similar way as for the mass according to

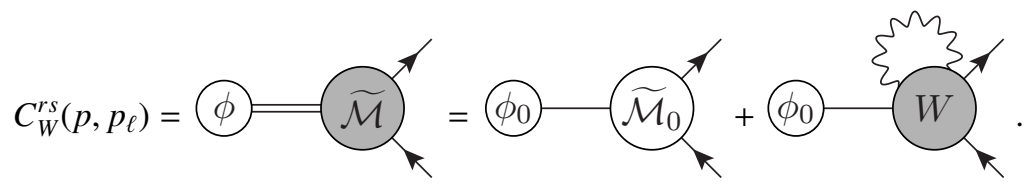

The grey blob containing $W$ is of order $e^{2}$ and can be separated, just like the Compton amplitude, into several irreducible vertex functions. The exact definitions of these vertex functions are quite involved and can be found in Ref. [2], but several comments can be made. First of all, the vertex functions are related to various structure-dependent form-factors containing both on-shell and off-shell information. Again, the off-shellness must cancel. The vertex functions also contain physical structure-dependent information (similar to how $\Gamma_{1}$ depends on the charge radius) and for $Y^{(2)}(L)$ this is the axial-vector form-factor $F_{A}\left(-m_{P}^{2}\right)=F_{A}^{P}$ from the real radiative decay $P^{-} \rightarrow \ell^{-} \bar{v}_{\ell} \gamma$.

By performing the amputation on the external meson leg in (12) to obtain the matrix element needed for the decay rate in (11), one finds the finite-volume function $Y^{(2)}(L)$ to be

$$
\begin{aligned}
Y^{(2)}(L) & =\frac{3}{4}+4 \log \left(\frac{m_{\ell}}{m_{W}}\right)+2 \log \left(\frac{m_{W} L}{4 \pi}\right)+\frac{c_{3}-2\left(c_{3}\left(\mathbf{v}_{\ell}\right)-B_{1}\left(\mathbf{v}_{\ell}\right)\right)}{2 \pi}- \\
& -2 A_{1}\left(\mathbf{v}_{\ell}\right)\left[\log \left(\frac{m_{P} L}{2 \pi}\right)+\log \left(\frac{m_{\ell} L}{2 \pi}\right)-1\right]-\frac{1}{m_{P} L}\left[\frac{\left(1+r_{\ell}^{2}\right)^{2} c_{2}-4 r_{\ell}^{2} c_{2}\left(\mathbf{v}_{\ell}\right)}{1-r_{\ell}^{4}}\right]+ \\
& +\frac{1}{\left(m_{P} L\right)^{2}}\left[-\frac{F_{A}^{P}}{f_{P}} \frac{4 \pi m_{P}\left[\left(1+r_{\ell}^{2}\right)^{2} c_{1}-4 r_{\ell}^{2} c_{1}\left(\mathbf{v}_{\ell}\right)\right]}{1-r_{\ell}^{4}}+\frac{8 \pi\left[\left(1+r_{\ell}^{2}\right) c_{1}-2 c_{1}\left(\mathbf{v}_{\ell}\right)\right]}{\left(1-r_{\ell}^{4}\right)}\right] .
\end{aligned}
$$

Here, $r_{\ell}=m_{\ell} / m_{P}, \mathbf{v}_{\ell}=\mathbf{p}_{\ell} / E_{\ell}$ the lepton velocity in terms of the energy $E_{\ell}$, and $m_{W}$ the $W$-boson mass. Also, $c_{k}, A_{1}\left(\mathbf{v}_{\ell}\right), B_{1}\left(\mathbf{v}_{\ell}\right)$ and $c_{j}\left(\mathbf{v}_{\ell}\right)$ are finite-volume coefficients defined in Ref. [2]. Note that no unphysical quantities appear. At order $1 / L^{2}$, there is one structuredependent contribution proportional to $F_{A}^{P}$ and the other term is purely point-like. This result is in perfect agreement with Ref. [4] for the universal terms up to $O(1 / L)$, which we derived in a completely different approach. The numerical impact of the $1 / L^{2}$-corrections is studied in Ref. [2].

\section{Conclusions}

We have presented a relativistic and model-independent method to derive electromagnetic FVEs beyond the point-like approximation. We are currently working to obtain the leading FVEs for semi-leptonic kaon decays, relevant for future precision tests in the SM flavour physics sector.

\section{Acknowledgements}

M.D.C, M.T.H., and A.P. are supported in part by UK STFC grant ST/P000630/1. Additionally M.T.H. is supported by UKRI Future Leader Fellowship MR/T019956/1. A.P. additionally received funding from the European Research Council (ERC) under the European Union's Horizon 2020 research and innovation programme under grant agreements No 757646 \& 813942 . N. H.-T. is funded by the Albert Einstein Center for Fundamental Physics at the University of Bern. 


\section{References}

[1] M. Hayakawa, S. Uno, Prog. Theor. Phys. 120, 413 (2008), 0804 . 2044

[2] M. Di Carlo, M.T. Hansen, N. Hermansson-Truedsson, A. Portelli (2021), 2109.05002

[3] S. Borsanyi et al., Science 347, 1452 (2015), 1406.4088

[4] V. Lubicz, G. Martinelli, C.T. Sachrajda, F. Sanfilippo, S. Simula, N. Tantalo, Phys. Rev. D95, 034504 (2017), 1611.08497

[5] P.D. Group, P.A. Zyla et al., Progress of Theoretical and Experimental Physics 2020 (2020), 083C01, https://academic.oup.com/ptep/article-pdf/2020/8/083C01/33653179/ptaa104.pdf

[6] Z. Davoudi, M.J. Savage, Phys. Rev. D90, 054503 (2014), 1402.6741

[7] N. Tantalo, V. Lubicz, G. Martinelli, C.T. Sachrajda, F. Sanfilippo, S. Simula, PoS LATTICE2016 (2016), 1612.00199

[8] N. Carrasco, V. Lubicz, G. Martinelli, C. Sachrajda, N. Tantalo, C. Tarantino, M. Testa, Phys. Rev. D 91, 074506 (2015), 1502.00257

[9] M. Di Carlo, D. Giusti, V. Lubicz, G. Martinelli, C. Sachrajda, F. Sanfilippo, S. Simula, N. Tantalo, Phys. Rev. D 100, 034514 (2019), 1904.08731 